\title{
New Results on Periodic Symbolic Sequences of Second Order Digital Filters with Two's Complement Arithmetic
}

\author{
Bingo Wing-Kuen Ling and Peter Kwong-Shun Tam \\ Department of Electronic and Information Engineering, The Hong Kong Polytechnic \\ University, Hung Hom, Kowloon, Hong Kong, China
}

\begin{abstract}
SUMMARY
In this article, the second order digital filter with two's complement arithmetic in [1] is considered. Necessary conditions for the symbolic sequences to be periodic after a number of iterations are given when the filter parameters are at $b=a+1$ and $b=-a+1$. Furthermore, for some particular values of $a$, even when one of the eigenvalues is outside the unit circle, the system may behave as a linear system after a number of iterations and the state vector may toggle between two states or converge to a fixed point at the steady state. The necessary and sufficient conditions for these phenomena are given in this article.
\end{abstract}

KEY WORDS: second order digital filter; two's complement arithmetic; symbolic sequences; eigenvalues 


\section{INTRODUCTION}

A nonlinear behavior may occur on a second order digital filter when the filter is implemented using a two's complement arithmetic for the addition operation. To analyze such a behavior, a symbolic analysis was proposed and the admissibility of the symbolic sequences was studied for the special case when $b=-1$ and $|a|<2[1-4,7]$. However, even when the symbolic sequence is admissible, there are many possibilities. In order to study the various possibilities, the set of admissible sequences can be partitioned into three subsets: One set contains periodic symbolic sequences. The second set contains symbolic sequences that are periodic after a number of iterations. The third set contains symbolic sequences that are never periodic. Some results on the first two sets were obtained for $b=-1$ and $|a|<2[1-4,7]$. These results have been extended for other real values of $a$, while the value of $b$ is still equal to $-1[5]$.

However, will those existing results remain valid if the filter parameter $b \neq-1$ ? Specifically, we are interested in the nonlinear behavior which may occur when $b=a+1$ and $b=-a+1$, and the answer to the following questions: Under what conditions will the symbolic sequence be periodic? If the symbolic sequence is periodic, under what conditions will the system behave as a linear system after a number of iterations and the state vector toggles among several states or converges to a fixed point? In this article, we focus on both the cases of $b=a+1$ and $b=-a+1$. In section 2, we will present the notations used in the existing literatures [1-7], and this article will also employ the same set of notations. In section 3, some new results on the above problems are presented. Finally, a conclusion is summarized in section 4. 


\section{NOTATIONS}

The notations used in [1-7] are adopted as follows:

The system is defined as:

$\left[\begin{array}{l}x_{1}(k+1) \\ x_{2}(k+1)\end{array}\right]=\mathrm{F}\left(\left[\begin{array}{l}x_{1}(k) \\ x_{2}(k)\end{array}\right]\right)=\left[\begin{array}{c}x_{2}(k) \\ f\left(b \cdot x_{1}(k)+a \cdot x_{2}(k)\right)\end{array}\right]=\mathbf{A} \cdot\left[\begin{array}{l}x_{1}(k) \\ x_{2}(k)\end{array}\right]+\mathbf{B} \cdot s_{k}$

where $\left[\begin{array}{l}x_{1}(k) \\ x_{2}(k)\end{array}\right] \in I^{2} \equiv\left\{\left(x_{1}, x_{2}\right):-1 \leq x_{1}<1,-1 \leq x_{2}<1\right\}$

$\mathbf{A}=\left[\begin{array}{ll}0 & 1 \\ b & a\end{array}\right]$

$\mathbf{B}=\left[\begin{array}{l}0 \\ 2\end{array}\right]$

$s_{k} \in\{-m, \cdots,-1,0,1, \cdots m\}$ where $m$ is the minimum integer satisfying

$-2 \cdot m-1 \leq b \cdot x_{1}+a \cdot x_{2}<2 \cdot m+1$

and $f(x)=x-2 \cdot n$ such that $2 \cdot n-1 \leq x<2 \cdot n+1$ and $n \in \mathrm{Z}^{+} \cup\{0\}$

Given an initial condition $\left[\begin{array}{l}x_{1}(0) \\ x_{2}(0)\end{array}\right] \in I^{2}$, a symbolic sequence $s=\left(s_{0}, s_{1}, \cdots\right) \in \sum$ can be generated by the map $S: I^{2} \rightarrow \sum$, and a sequence $s$ in $\sum$ is admissible if $\exists\left[\begin{array}{l}x_{1}(0) \\ x_{2}(0)\end{array}\right] \in I^{2}$ such that $\mathrm{S}\left(\left[\begin{array}{l}x_{1}(0) \\ x_{2}(0)\end{array}\right]\right)=s$.

The set $\sum$ can be partitioned into three subsets: $\Sigma_{\alpha}=\left\{s=\left(s_{0} s_{1} s_{2} \cdots\right): s\right.$ is periodic $\}$, $\Sigma_{\beta}=\left\{s=\left(s_{0} s_{1} s_{2} \cdots\right): s\right.$ is periodic after a number of iterations $\} \quad$ and $\Sigma_{\gamma}=\Sigma \backslash\left(\Sigma_{\alpha} \cup \Sigma_{\beta}\right)$ 


\section{NEW RESULTS FOR THE PERIODIC SYMBOLIC SEQUENCES}

This section presents several conditions for the state vector to be periodic after a number of iterations. These conditions can be stated in the following lemmas, theorems and remarks, where the stability of these periodic orbits is stated in the observations:

\section{Lemma 1}

For $b=a+1$, if $\exists M \in Z^{+}$such that $\left[\begin{array}{l}x_{1}(k) \\ x_{2}(k)\end{array}\right]=\left[\begin{array}{l}x_{1}(k+M) \\ x_{2}(k+M)\end{array}\right]$ for $k \geq k_{0}$, then $\left(x_{1}(0)+x_{2}(0)\right) \cdot\left((a+1)^{M}-1\right)+2 \cdot\left(2 \cdot(a+1)^{M}-1\right) \cdot \sum_{j=0}^{k_{0}-1} \frac{s_{j}}{(a+1)^{j+1}}+2 \cdot(a+1)^{M} \cdot \sum_{j=k_{0}}^{k_{0}+M-1} \frac{s_{j}}{(a+1)^{j+1}}=0$.

\section{Proof}

For $b=a+1$, the nonlinear system in (1) can be represented by the state equation $\left[\begin{array}{l}x_{1}(k+1) \\ x_{2}(k+1)\end{array}\right]=\left[\begin{array}{cc}0 & 1 \\ a+1 & a\end{array}\right] \cdot\left[\begin{array}{l}x_{1}(k) \\ x_{2}(k)\end{array}\right]+\left[\begin{array}{l}0 \\ 2\end{array}\right] \cdot s_{k}$. Hence, the solution of the system is:

$\left[\begin{array}{l}x_{1}(k) \\ x_{2}(k)\end{array}\right]=\frac{1}{a+2} \cdot\left[\begin{array}{cc}(a+1)^{k}+(a+1) \cdot(-1)^{k} & (a+1)^{k}-(-1)^{k} \\ (a+1)^{k+1}-(a+1) \cdot(-1)^{k} & (a+1)^{k+1}+(-1)^{k}\end{array}\right] \cdot\left[\begin{array}{c}x_{1}(0) \\ x_{2}(0)\end{array}\right]+\frac{2}{a+2} \cdot \sum_{j=0}^{k-1}\left[\begin{array}{c}(a+1)^{k-1-j}-(-1)^{k-1-j} \\ (a+1)^{k-j}+(-1)^{k-1-j}\end{array}\right] \cdot s_{j}$

If $\exists M \in \mathrm{Z}^{+}$such that $\left[\begin{array}{l}x_{1}(k) \\ x_{2}(k)\end{array}\right]=\left[\begin{array}{l}x_{1}(k+M) \\ x_{2}(k+M)\end{array}\right]$ for $k \geq k_{0}$, then:

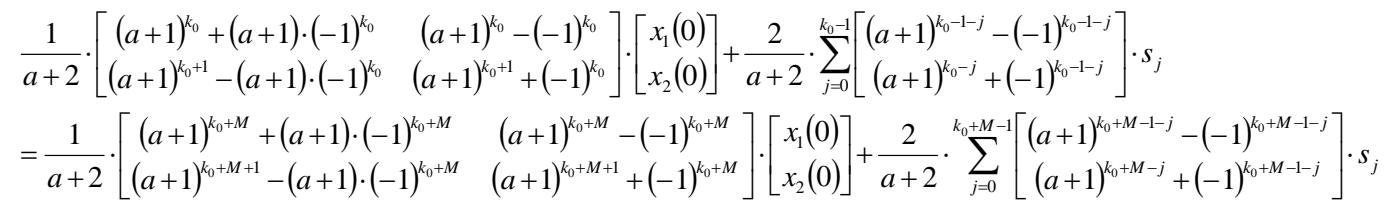

Hence,

$$
\begin{aligned}
& \left\{\begin{array}{l}
\left((a+1)^{M}-1\right) \cdot \frac{(a+1)^{k_{0}}}{a+2} \cdot\left(x_{1}(0)+x_{2}(0)+2 \cdot \sum_{j=0}^{k_{0}-1} \frac{s_{j}}{(a+1)^{j+1}}\right)+\left((-1)^{M}-1\right) \cdot \frac{(-1)^{k_{0}}}{a+2} \cdot\left((a+1) \cdot x_{1}(0)-x_{2}(0)-2 \cdot \sum_{j=0}^{k_{0}-1} \frac{s_{j}}{(-1)^{j+1}}\right) \\
+\frac{2}{a+2} \cdot \sum_{j=0}^{k_{0}+M-1} s_{j} \cdot\left((a+1)^{k_{0}+M-1-j}-(-1)^{k_{0}+M-1-j}\right)=0
\end{array}\right. \\
& \left\{\begin{array}{l}
\left((a+1)^{M}-1\right) \cdot \frac{(a+1)^{k_{0}+1}}{a+2} \cdot\left(x_{1}(0)+x_{2}(0)+2 \cdot \sum_{j=0}^{k_{0}-1} \frac{s_{j}}{(a+1)^{j+1}}\right)+\left((-1)^{M}-1\right) \cdot \frac{(-1)^{k_{0}}}{a+2} \cdot\left(2 \cdot \sum_{j=0}^{k_{0}-1} \frac{s_{j}}{(-1)^{j+1}}-\left((a+1) \cdot x_{1}(0)-x_{2}(0)\right)\right)(10) \\
+\frac{2}{a+2} \cdot \sum_{j=0}^{k_{0}+M-1} s_{j} \cdot\left((a+1)^{k_{0}+M-j}+(-1)^{k_{0}+M-1-j}\right)=0
\end{array}\right.
\end{aligned}
$$


Let

$$
\begin{aligned}
& t_{1}=\left((a+1)^{M}-1\right) \cdot \frac{(a+1)^{k_{0}}}{a+2} \cdot\left(x_{1}(0)+x_{2}(0)+2 \cdot \sum_{j=0}^{k_{0}-1} \frac{s_{j}}{(a+1)^{j+1}}\right) \\
& t_{2}=\left((-1)^{M}-1\right) \cdot \frac{(-1)^{k_{0}}}{a+2} \cdot\left((a+1) \cdot x_{1}(0)-x_{2}(0)-2 \cdot \sum_{j=0}^{k_{0}-1} \frac{s_{j}}{(-1)^{j+1}}\right) \\
& t_{3}=\frac{2}{a+2} \cdot \sum_{j=0}^{k_{0}+M-1} s_{j} \cdot(a+1)^{k_{0}+M-1-j} \\
& t_{4}=\frac{2}{a+2} \cdot \sum_{j=0}^{k_{0}+M-1} s_{j} \cdot(-1)^{k_{0}+M-1-j}
\end{aligned}
$$

Then we have:

$\left\{\begin{array}{l}t_{1}+t_{2}+t_{3}-t_{4}=0 \\ (a+1) \cdot t_{1}-t_{2}+(a+1) \cdot t_{3}+t_{4}=0\end{array}\right.$

which

$$
\Rightarrow a=-2 \text { or } t_{1}=-t_{3}
$$

For $a \neq-2$, we have $t_{1}=-t_{3}$, that is:

$$
\begin{aligned}
& \left((a+1)^{M}-1\right) \cdot \frac{(a+1)^{k_{0}}}{a+2} \cdot\left(x_{1}(0)+x_{2}(0)+2 \cdot \sum_{j=0}^{k_{0}-1} \frac{s_{j}}{(a+1)^{j+1}}\right)=-\frac{2}{a+2} \cdot \sum_{j=0}^{k_{0}+M-1} s_{j} \cdot(a+1)^{k_{0}+M-1-j} \\
& \Rightarrow\left(x_{1}(0)+x_{2}(0)\right) \cdot\left((a+1)^{M}-1\right)+2 \cdot\left(2 \cdot(a+1)^{M}-1\right) \cdot \sum_{j=0}^{k_{0}-1} \frac{s_{j}}{(a+1)^{j+1}}+2 \cdot(a+1)^{M} \cdot \sum_{j=k_{0}}^{k_{0}+M-1} \frac{s_{j}}{(a+1)^{j+1}}=0
\end{aligned}
$$

\section{$\underline{\text { Remark } 1}$}

If, after a number of iterations, the state vector is periodic with period $M$, then the symbolic sequence will also be periodic with the same period, that is, $s \in \Sigma_{\beta}$. Hence, this lemma gives a necessary condition for a symbolic sequence to be periodic after a number of iterations.

However, $s_{k}$ is an integer in $\{-m, \cdots,-1,0,1, \cdots m\}$ and the periodicity of the symbolic sequence is $M$. So there are $(2 \cdot m+1)^{M}$ possibilities. In the following 
theorem, the case of $s_{k}=0$ for $k \geq k_{0}$ is discussed.

\section{Theorem 1}

For $b=a+1$ and $|a+1|>1, \quad s_{k}=0$ for $k \geq k_{0}$ if and only if $\exists k_{0} \in Z^{+} \bigcup\{0\}$ such that $x_{1}\left(k_{0}\right)=-x_{2}\left(k_{0}\right)$.

\section{$\underline{\text { Proof }}$}

For the if part, since $b=a+1$ and $s_{k}=0$ for $k \geq k_{0}$, we have:

$\left[\begin{array}{l}x_{1}(k) \\ x_{2}(k)\end{array}\right]=\frac{1}{a+2} \cdot\left[\begin{array}{c}(a+1)^{k-k_{0}} \cdot\left(x_{1}\left(k_{0}\right)+x_{2}\left(k_{0}\right)\right)+(-1)^{k-k_{0}} \cdot\left((a+1) \cdot x_{1}\left(k_{0}\right)-x_{2}\left(k_{0}\right)\right) \\ (a+1)^{k-k_{0}+1} \cdot\left(x_{1}\left(k_{0}\right)+x_{2}\left(k_{0}\right)\right)-(-1)^{k-k_{0}} \cdot\left((a+1) \cdot x_{1}\left(k_{0}\right)-x_{2}\left(k_{0}\right)\right)\end{array}\right]$

Since $\quad|a+1|>1, \quad(a+1)^{k-k_{0}} \cdot\left(x_{1}\left(k_{0}\right)+x_{2}\left(k_{0}\right)\right) \quad$ diverges $\quad$ as $\quad k \rightarrow+\infty \quad$ if $x_{1}\left(k_{0}\right)+x_{2}\left(k_{0}\right) \neq 0$. However, as $s_{k}=0$ for $k \geq k_{0}$, this implies that $\left[\begin{array}{l}x_{1}(k) \\ x_{2}(k)\end{array}\right] \in I^{2}$ and that $(a+1)^{k-k_{0}} \cdot\left(x_{1}\left(k_{0}\right)+x_{2}\left(k_{0}\right)\right)$ is bounded for $k \geq k_{0}$. Hence, $x_{1}\left(k_{0}\right)+x_{2}\left(k_{0}\right)=0$ and this proves the if part.

For the only if part, since $x_{1}\left(k_{0}\right)=-x_{2}\left(k_{0}\right)$, we have:

$$
\begin{aligned}
& {\left[\begin{array}{l}
x_{1}\left(k_{0}+1\right) \\
x_{2}\left(k_{0}+1\right)
\end{array}\right]=\left[\begin{array}{c}
-x_{1}\left(k_{0}\right) \\
f\left((a+1) \cdot x_{1}\left(k_{0}\right)-a \cdot x_{1}\left(k_{0}\right)\right)
\end{array}\right]=\left[\begin{array}{c}
-x_{1}\left(k_{0}\right) \\
f\left(x_{1}\left(k_{0}\right)\right)
\end{array}\right] \text {. Since }\left|x_{1}\left(k_{0}\right)\right|<1 \text {, we have }} \\
& {\left[\begin{array}{l}
x_{1}\left(k_{0}+1\right) \\
x_{2}\left(k_{0}+1\right)
\end{array}\right]=\left[\begin{array}{c}
-x_{1}\left(k_{0}\right) \\
x_{1}\left(k_{0}\right)
\end{array}\right] \text { and } s_{k_{0}}=0 .}
\end{aligned}
$$

Similarly, we have $\quad\left[\begin{array}{l}x_{1}\left(k_{0}+2\right) \\ x_{2}\left(k_{0}+2\right)\end{array}\right]=\left[\begin{array}{c}x_{1}\left(k_{0}\right) \\ -x_{1}\left(k_{0}\right)\end{array}\right] \quad$ and $\quad s_{k_{0}+1}=0 \quad$. Since $\left[\begin{array}{l}x_{1}\left(k_{0}+2\right) \\ x_{2}\left(k_{0}+2\right)\end{array}\right]=\left[\begin{array}{l}x_{1}\left(k_{0}\right) \\ x_{2}\left(k_{0}\right)\end{array}\right]$, we have $s_{k}=0$ for $k \geq k_{0}$ and this proves the only if part.

\section{$\underline{\text { Remark } 2}$}

The eigenvalues of matrix $\mathbf{A}$ is -1 and $a+1$. Since $|a+1|>1$, one of the 
eigenvalues is outside the unit circle. However, the system may behave as a linear system when $s_{k}=0$ for $k \geq k_{0}$. Theorem 1 gives the necessary and sufficient condition for the nonlinear system to behave as a linear system after a number of iterations. It is interesting to note that the system may behave as a linear system after a number of iterations if and only if the state vector toggles between two points on a particular straight line of the phase portrait.

\section{$\underline{\text { Example 1 }}$}

Consider the system $\left[\begin{array}{l}x_{1}(k+1) \\ x_{2}(k+1)\end{array}\right]=\mathbf{A} \cdot\left[\begin{array}{l}x_{1}(k) \\ x_{2}(k)\end{array}\right]+\mathbf{B} \cdot s_{k}$, where $\mathbf{A}=\left[\begin{array}{cc}0 & 1 \\ a+1 & a\end{array}\right], \quad \mathbf{B}=\left[\begin{array}{l}0 \\ 2\end{array}\right]$, $a=3$ and $\left[\begin{array}{l}x_{1}(0) \\ x_{2}(0)\end{array}\right]=\left[\begin{array}{c}0.9003 \\ -0.5377\end{array}\right]$. Figure 1 shows the phase portrait of the system. It can be seen from the figure that the state vector toggles between two states at the steady state on a particular straight line $x_{1}=-x_{2}$ of the phase portrait.

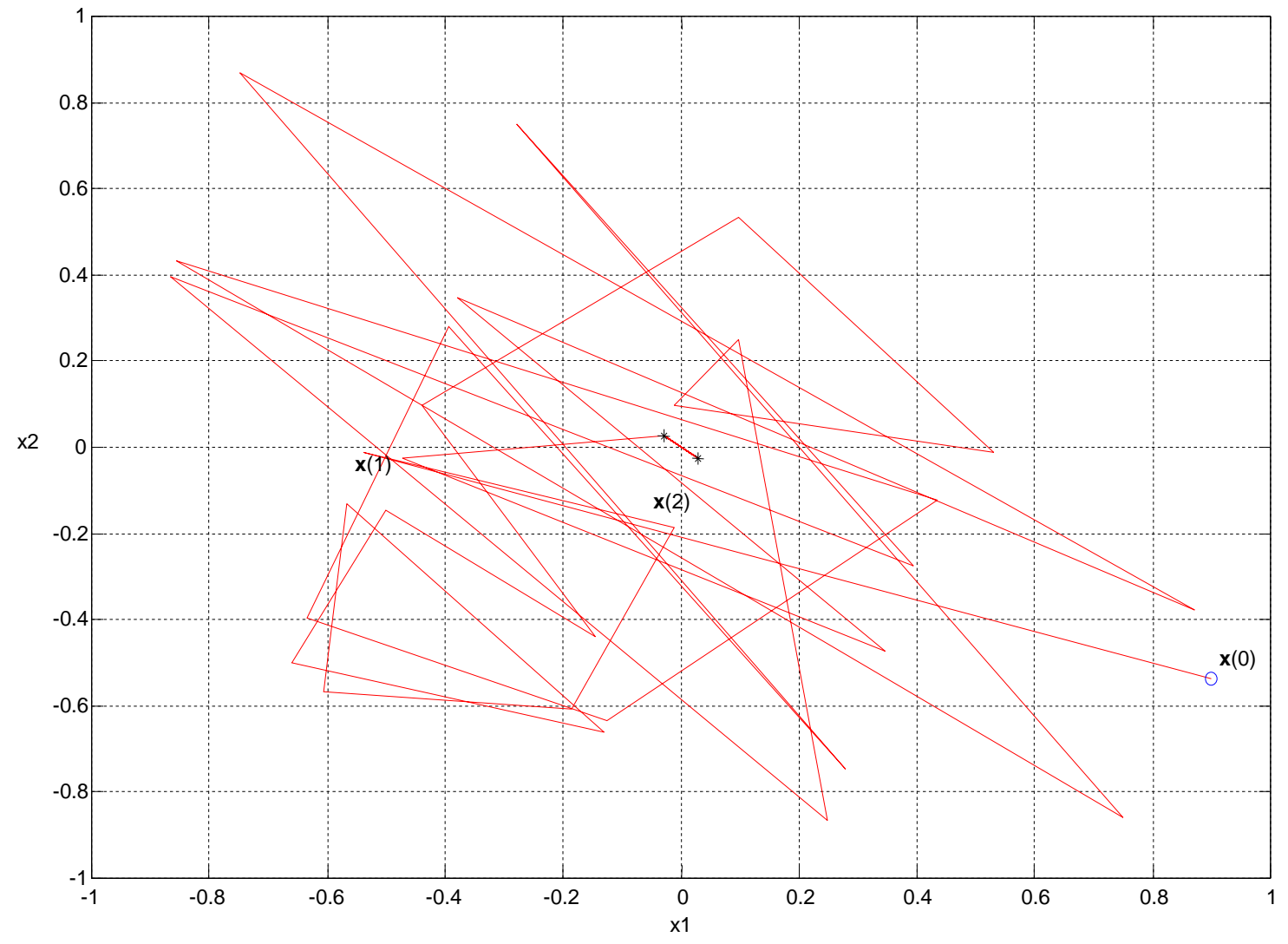

Figure 1. The phase portrait of the second order digital filter with two's complement arithmetic. The points $\mathbf{x}(0), \mathbf{x}(1), \mathbf{x}(2)$ are as annotated, and the points with '*' denote the 'steady states' of $\mathbf{x}$. 
We have discussed the case when $s_{k}=0$ for $k \geq k_{0}$ in theorem 1. What happens when $s_{k} \neq 0$ for $k \geq k_{0}$ ? We will present an interesting result in the following theorem:

\section{Theorem 2}

For $b=a+1$ and $a$ being an odd integer, $\exists k_{0} \in Z^{+} \cup\{0\}$ such that $x_{1}\left(k_{0}\right)=x_{2}\left(k_{0}\right)=-1$ if and only if $s_{k}=a$ and $\left[\begin{array}{l}x_{1}(k) \\ x_{2}(k)\end{array}\right]=\left[\begin{array}{l}x_{1}\left(k_{0}\right) \\ x_{2}\left(k_{0}\right)\end{array}\right]$ for $k \geq k_{0}$.

\section{$\underline{\text { Proof }}$}

For the if part, since $\left[\begin{array}{l}x_{1}\left(k_{0}+1\right) \\ x_{2}\left(k_{0}+1\right)\end{array}\right]=\left[\begin{array}{c}x_{2}\left(k_{0}\right) \\ f\left((a+1) \cdot x_{1}\left(k_{0}\right)+a \cdot x_{2}\left(k_{0}\right)\right)\end{array}\right]$, if $\exists k_{0} \in \mathrm{Z}^{+} \bigcup\{0\}$ such that $x_{1}\left(k_{0}\right)=x_{2}\left(k_{0}\right)=-1$, then we have $\left[\begin{array}{l}x_{1}\left(k_{0}+1\right) \\ x_{2}\left(k_{0}+1\right)\end{array}\right]=\left[\begin{array}{c}-1 \\ f(-(2 \cdot a+1))\end{array}\right]$. Since $a$ is an odd integer, we have $\left[\begin{array}{l}x_{1}\left(k_{0}+1\right) \\ x_{2}\left(k_{0}+1\right)\end{array}\right]=\left[\begin{array}{l}-1 \\ -1\end{array}\right]$ and $\quad s_{k_{0}}=a$. As $\left[\begin{array}{l}x_{1}\left(k_{0}+1\right) \\ x_{2}\left(k_{0}+1\right)\end{array}\right]=\left[\begin{array}{l}x_{1}\left(k_{0}\right) \\ x_{2}\left(k_{0}\right)\end{array}\right]=\left[\begin{array}{l}-1 \\ -1\end{array}\right]$, we have $s_{k}=a$ and $x_{1}(k)=x_{2}(k)=-1$ for $k \geq k_{0}$. For the only if part, since $\left[\begin{array}{l}x_{1}\left(k_{0}+1\right) \\ x_{2}\left(k_{0}+1\right)\end{array}\right]=\left[\begin{array}{c}x_{2}\left(k_{0}\right) \\ f\left((a+1) \cdot x_{1}\left(k_{0}\right)+a \cdot x_{2}\left(k_{0}\right)\right)\end{array}\right], \quad$ if $x_{1}(k)=x_{1}\left(k_{0}\right) \quad$ and $\quad x_{2}(k)=x_{2}\left(k_{0}\right) \quad$ for $\quad k \geq k_{0}, \quad$ then we have $\left[\begin{array}{l}x_{1}\left(k_{0}\right) \\ x_{2}\left(k_{0}\right)\end{array}\right]=\left[\begin{array}{c}x_{2}\left(k_{0}\right) \\ f\left((a+1) \cdot x_{1}\left(k_{0}\right)+a \cdot x_{2}\left(k_{0}\right)\right)\end{array}\right]$, which implies $x_{1}\left(k_{0}\right)=x_{2}\left(k_{0}\right) \quad$ and $x_{2}\left(k_{0}\right)=f\left((a+1) \cdot x_{1}\left(k_{0}\right)+a \cdot x_{2}\left(k_{0}\right)\right)$. Since $s_{k}=a$ for $k \geq k_{0}$, we have $x_{1}\left(k_{0}\right)=(a+1) \cdot x_{1}\left(k_{0}\right)+a \cdot x_{1}\left(k_{0}\right)+2 \cdot a$, which implies $2 \cdot a \cdot\left(x_{1}\left(k_{0}\right)+1\right)=0$. As $a$ is an odd integer, so $a \neq 0$. As a result, we have $x_{1}\left(k_{0}\right)=-1$ and we prove the only if part.

\section{$\underline{\text { Remark } 3}$}


Theorem 2 states the necessary and sufficient condition for the state vector to stay at a fixed point after a number of iterations when the parameter $a$ is an odd integer. It is interesting to note that this fixed point is $(-1,-1)$.

\section{Example 2}

Consider the system $\left[\begin{array}{l}x_{1}(k+1) \\ x_{2}(k+1)\end{array}\right]=\mathbf{A} \cdot\left[\begin{array}{l}x_{1}(k) \\ x_{2}(k)\end{array}\right]+\mathbf{B} \cdot s_{k}$, where $\mathbf{A}=\left[\begin{array}{cc}0 & 1 \\ a+1 & a\end{array}\right], \quad \mathbf{B}=\left[\begin{array}{l}0 \\ 2\end{array}\right]$, $a=3$ and $\left[\begin{array}{l}x_{1}(0) \\ x_{2}(0)\end{array}\right]=\left[\begin{array}{c}0.1875 \\ -0.25\end{array}\right]$. Figure 2 shows the phase portrait of the system. It can be seen from the figure that the state vector converges to a fixed point $\left[\begin{array}{l}x_{1}(k) \\ x_{2}(k)\end{array}\right]=\left[\begin{array}{l}-1 \\ -1\end{array}\right]$

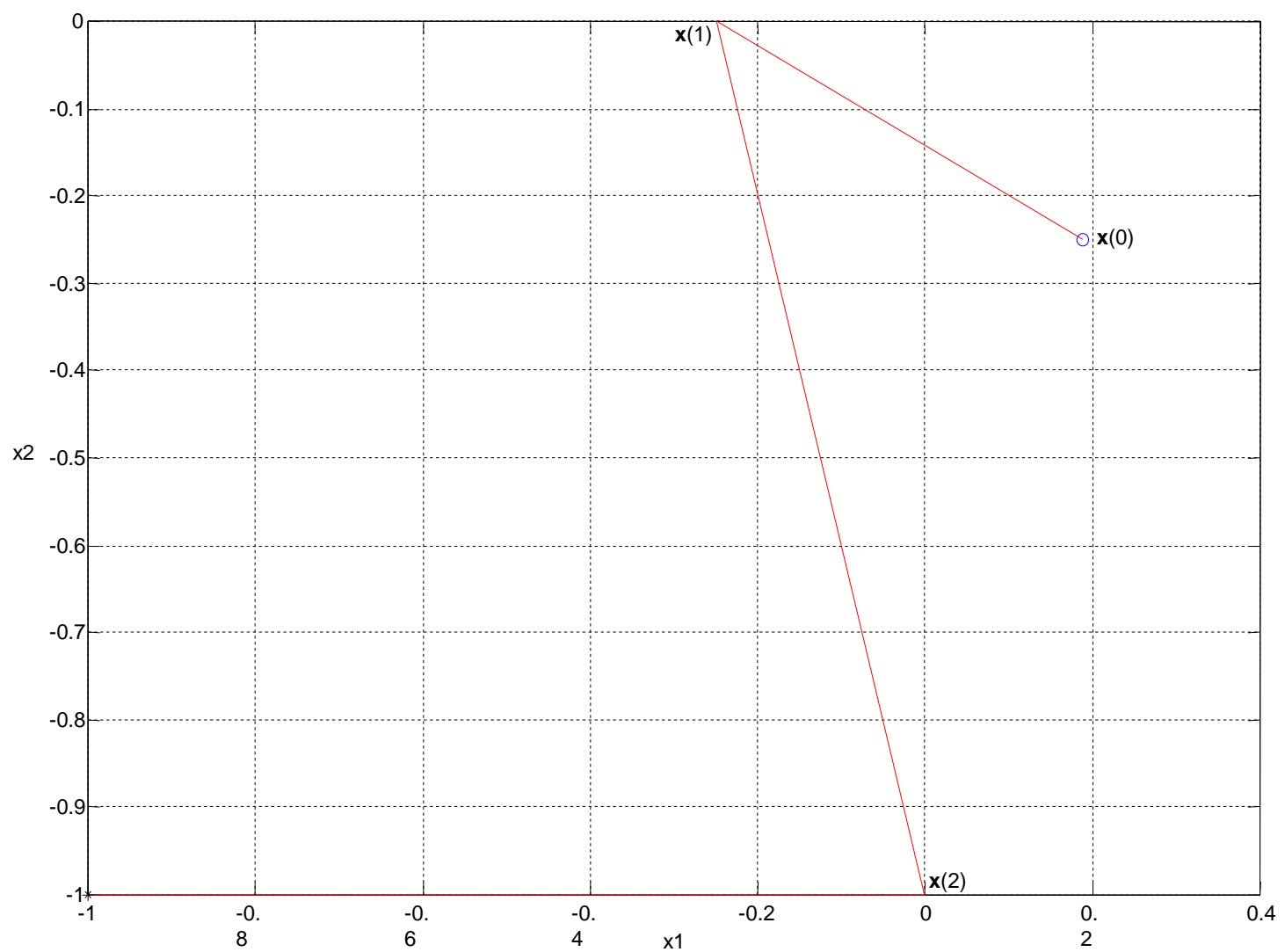

Figure 2. The phase portrait of the second order digital filter with two's complement arithmetic. The points $\mathbf{x}(0), \mathbf{x}(1), \mathbf{x}(2)$ are as annotated, and the point with '*' denotes the 'steady state' of $\mathbf{x}$.

\section{Remark 4}

The above results are obtained when $b=a+1$. What happens when $b=-a+1$ ? We can show that results similar to lemma 1 and theorem 1 can be obtained as lemma 2 
and theorem 3, while the results of theorem 2 can be modified to that of theorem 4 .

\section{$\underline{\text { Remark } 5}$}

Since one of the eigenvalues is unstable, one may predict that the periodic orbits are unstable. However, a counter-intuitive result is found that the periodic orbits are stable if $a$ is an odd integer. The result is stated in observation 1 below:

\section{Observation 1}

When $b=a+1$ and $a$ is an odd integer, the state vector toggles between two states at the steady state on a particular straight line $x_{1}=-x_{2}$ of the phase portrait or converges to a fixed point $\mathbf{x}^{*}=\left[\begin{array}{l}-1 \\ -1\end{array}\right]$ for all the initial conditions in $I^{2}$.

To demonstrate this phenomenon, a random initial condition $\mathbf{x}(0)$ is generated in $I^{2}$, it can be shown in figure 3 a that the state converges to a period-2 signal.

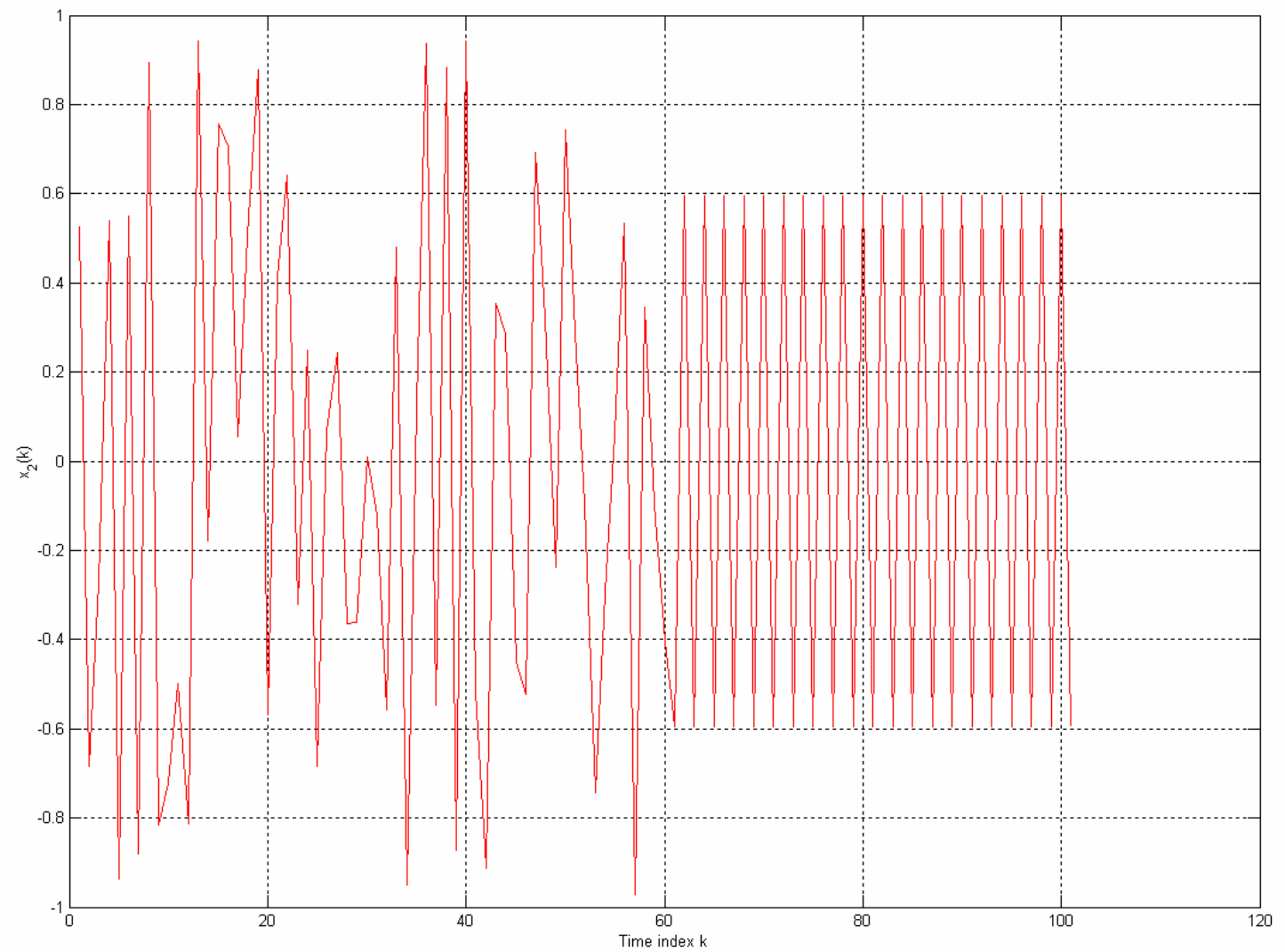

Figure 3a. The phase portrait of the second order digital filter with two's complement arithmetic. The initial condition $\mathbf{x}(0)=\left[\begin{array}{lll}0.7826 & 0.5242\end{array}\right]^{\mathrm{T}}$ is generated randomly. When $a=5$ and $b=6$, the state converges to a period- 2 signal. 
However, when a deviates from an odd integer a little bit, the state neither converges to a periodic signal nor a fixed point, as shown in figure $3 \mathrm{~b}$.

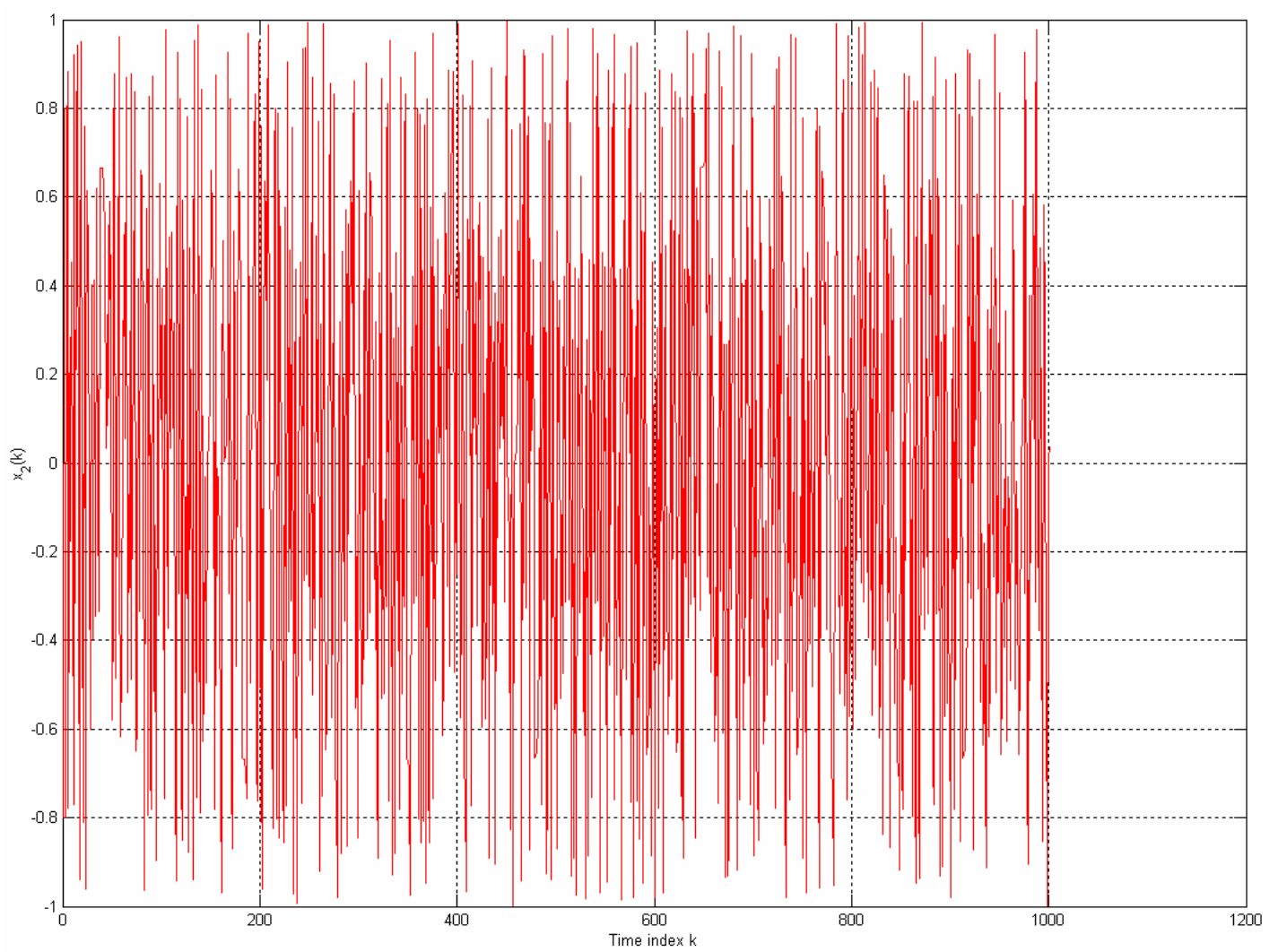

Figure $3 \mathrm{~b}$. The phase portrait of the second order digital filter with two's complement arithmetic. The initial condition is $\mathbf{x}(0)=[0.8-0.7999]^{\mathrm{T}}, a=3.001$ and $b=4.001$, the state neither converges to a periodic signal nor a fixed point.

\section{Lemma 2}

For $b=-a+1$, if $\exists M \in Z^{+}$such that $\left[\begin{array}{l}x_{1}(k) \\ x_{2}(k)\end{array}\right]=\left[\begin{array}{l}x_{1}(k+M) \\ x_{2}(k+M)\end{array}\right]$ for $k \geq k_{0}$, then $\left(1-(a-1)^{M}\right) \cdot\left(x_{1}(0)-x_{2}(0)-2 \cdot \sum_{j=0}^{k_{0}-1} \frac{s_{j}}{(a-1)^{j+1}}\right)+2 \cdot \sum_{j=k_{0}}^{k_{0}+M-1}(a+1)^{M-j-1} \cdot s_{j}=0$.

\section{Theorem 3}

For $b=-a+1$ and $|a-1|>1, s_{k}=0$ for $k \geq k_{0}$ if and only if $\exists k_{0} \in Z^{+} \bigcup\{0\}$ such that $x_{1}\left(k_{0}\right)=x_{2}\left(k_{0}\right)$.

\section{Example 3}

Consider the system $\left[\begin{array}{l}x_{1}(k+1) \\ x_{2}(k+1)\end{array}\right]=\mathbf{A} \cdot\left[\begin{array}{l}x_{1}(k) \\ x_{2}(k)\end{array}\right]+\mathbf{B} \cdot s_{k}, \quad$ where $\quad \mathbf{A}=\left[\begin{array}{cc}0 & 1 \\ -a+1 & a\end{array}\right]$, 
$\mathbf{B}=\left[\begin{array}{l}0 \\ 2\end{array}\right], a=3$ and $\left[\begin{array}{l}x_{1}(0) \\ x_{2}(0)\end{array}\right]=\left[\begin{array}{l}-0.1875 \\ -0.1234\end{array}\right]$. Figure 4 shows the phase portrait of the system. It can be seen from the figure that the state vector converges to a fixed point on a particular straight line $x_{1}=x_{2}$ of the phase portrait.

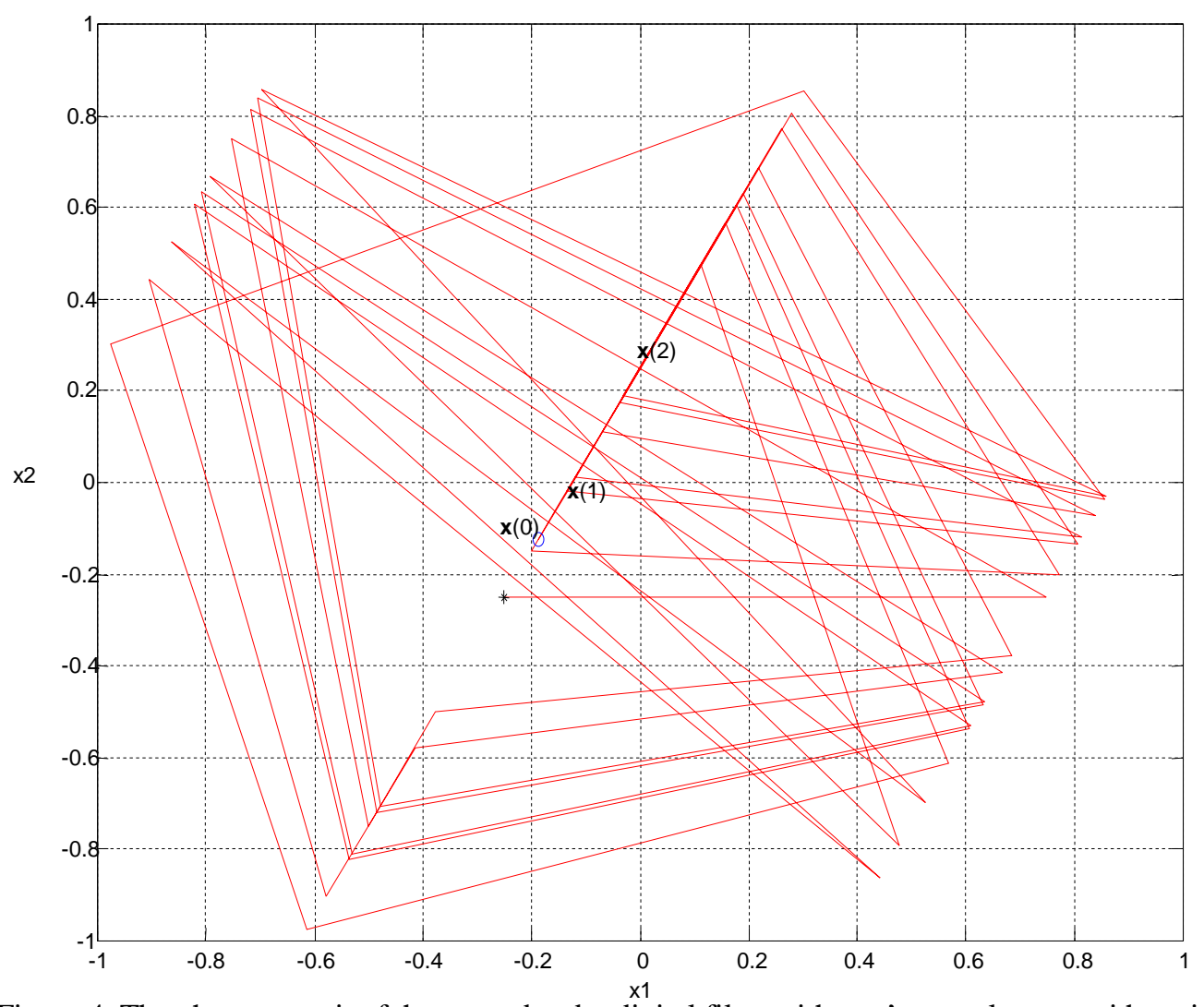

Figure 4. The phase portrait of the second order digital filter with two's complement arithmetic. The points $\mathbf{x}(0), \mathbf{x}(1), \mathbf{x}(2)$ are as annotated, and the point with '*' denotes the 'steady state' of $\mathbf{x}$.

\section{Theorem 4}

For $b=-a+1$ and $a$ being an odd integer, there does not exist $k_{0} \in \mathrm{Z}^{+}$such that $s_{k}=a$ for $k \geq k_{0}$.

\section{$\underline{\text { Proof }}$}

Since $\left[\begin{array}{l}x_{1}(k) \\ x_{2}(k)\end{array}\right]=\mathbf{A}^{k-k_{0}} \cdot\left[\begin{array}{l}x_{1}\left(k_{0}\right) \\ x_{2}\left(k_{0}\right)\end{array}\right]+\sum_{j=k_{0}}^{k-1} \mathbf{A}^{k-1-j} \cdot \mathbf{B} \cdot s_{j}$ for $k>k_{0}$, if $s_{k}=a$ for $k \geq k_{0}$,

we have:

$\left[\begin{array}{l}x_{1}(k) \\ x_{2}(k)\end{array}\right]=\frac{1}{a-2} \cdot\left[\begin{array}{c}(a-1)^{k-k_{0}} \cdot\left(x_{2}\left(k_{0}\right)-x_{1}\left(k_{0}\right)-\frac{2 \cdot a}{2-a}\right)+(a-1) \cdot x_{1}\left(k_{0}\right)-x_{2}\left(k_{0}\right)+\frac{2 \cdot a}{2-a}-2 \cdot a \cdot\left(k-k_{0}\right) \\ (a-1)^{k-k_{0}+1} \cdot\left(x_{2}\left(k_{0}\right)-x_{1}\left(k_{0}\right)-\frac{2 \cdot a}{2-a}\right)+(a-1) \cdot x_{1}\left(k_{0}\right)-x_{2}\left(k_{0}\right)+\frac{2 \cdot a \cdot(a-1)}{2-a}-2 \cdot a \cdot\left(k-k_{0}\right)\end{array}\right]$ 
As $k \rightarrow+\infty, k-k_{0} \rightarrow+\infty$, so $\lim _{k \rightarrow+\infty}\left[\begin{array}{l}x_{1}(k) \\ x_{2}(k)\end{array}\right] \notin I^{2}$. Hence, there does not exist $k_{0} \in \mathrm{Z}^{+}$such that $s_{k}=a$ for $k \geq k_{0}$, and this proves the theorem.

\section{Example 4}

Consider the system $\left[\begin{array}{l}x_{1}(k+1) \\ x_{2}(k+1)\end{array}\right]=\mathbf{A} \cdot\left[\begin{array}{l}x_{1}(k) \\ x_{2}(k)\end{array}\right]+\mathbf{B} \cdot s_{k}, \quad$ where $\quad \mathbf{A}=\left[\begin{array}{cc}0 & 1 \\ -a+1 & a\end{array}\right]$, $\mathbf{B}=\left[\begin{array}{l}0 \\ 2\end{array}\right], \quad a=3$ and $\left[\begin{array}{l}x_{1}(0) \\ x_{2}(0)\end{array}\right]=\left[\begin{array}{l}0.7826 \\ 0.5242\end{array}\right]$. Figure 5 shows the phase portrait of the system.

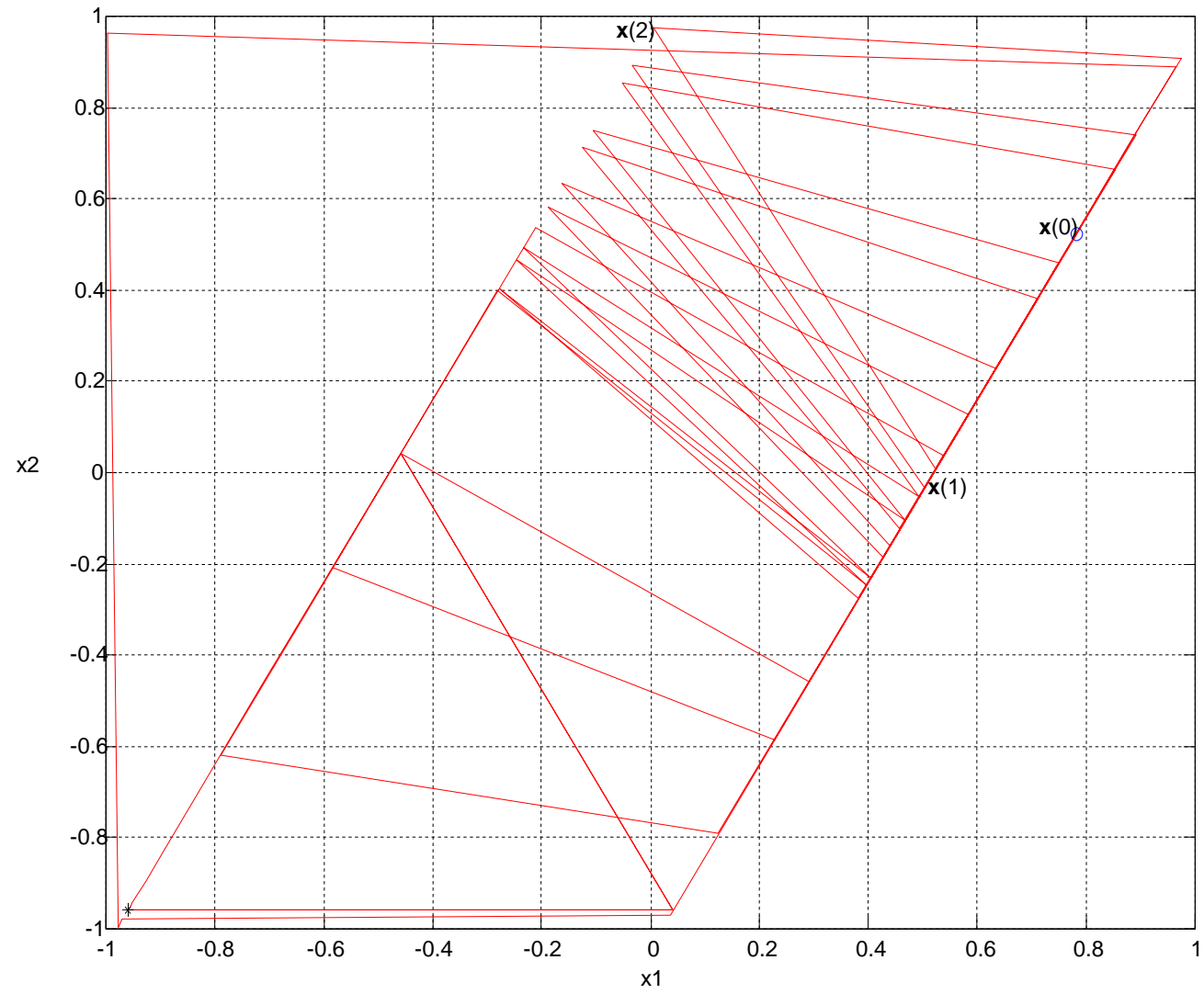

Figure 5. The phase portrait of the second order digital filter with two's complement arithmetic. The points $\mathbf{x}(0), \mathbf{x}(1), \mathbf{x}(2)$ are as annotated, and the point with '*' denotes the 'steady state' of $\mathbf{x}$.

\section{Observation 2}

When $b=-a+1$ and $a$ is an odd integer, the state vector converges to a fixed point on a particular straight line $x_{1}=x_{2}$ of the phase portrait for all the initial conditions in $I^{2}$. 
To demonstrate this phenomenon, a random initial condition $\mathbf{x}(0)$ is generated in $I^{2}$, it can be shown in figure 6 a that the state converges to a fixed point.

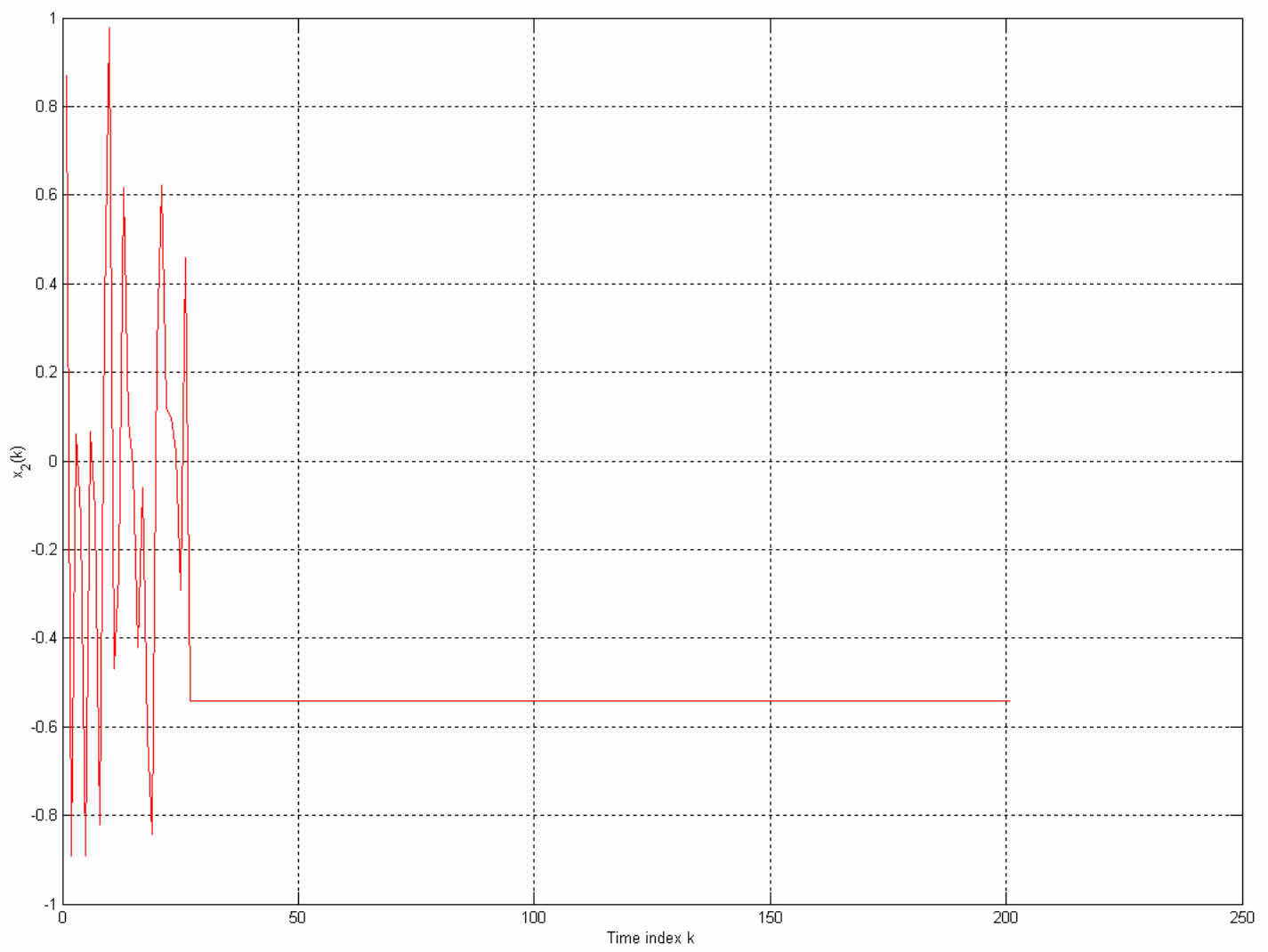

Figure 6a. The phase portrait of the second order digital filter with two's complement arithmetic. The initial condition is $\mathbf{x}(0)=\left[\begin{array}{lll}-0.1886 & 0.87909\end{array}\right]^{\mathrm{T}}, a=5$ and $b=-4$, the state converges to a fixed point.

However, when a deviates from an odd integer a little bit, the state does not converge to a fixed point, as shown in figure $6 \mathrm{~b}$. 


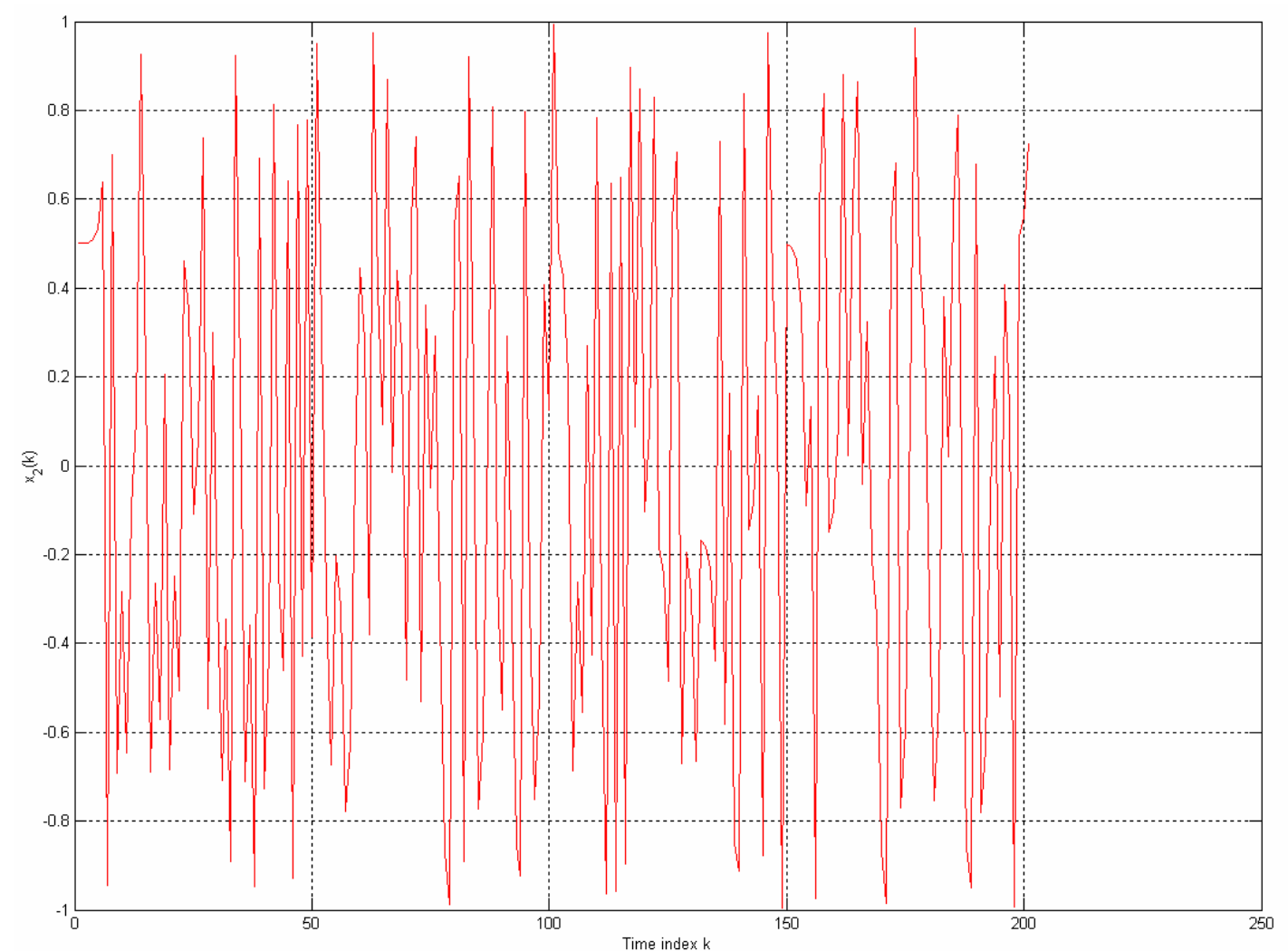

Figure 6b. The phase portrait of the second order digital filter with two's complement arithmetic. The initial condition is $\mathbf{x}(0)=\left[\begin{array}{ll}0.5 & 0.5001\end{array}\right]^{\mathrm{T}}, a=5.01$ and $b=-4.01$, the state does not converge to a fixed point. 


\section{CONCLUSIONS}

In this article, some interesting behaviors of second-order digital filters with two's complement arithmetic are explored. The cases of $b=a+1$ and $b=-a+1$ are analyzed and some necessary conditions for the symbolic sequences to be periodic after a number of iterations are given. The necessary and sufficient conditions for the system to behave as a linear system after a number of iterations and the state vector to toggle among several states or converge to a fixed point are given. 


\section{ACKNOWLEDGEMENT}

The work described in this article was substantially supported by The Hong Kong Polytechnic University. 


\section{REFERENCES}

[1] Chua LO, Lin T. Chaos in digital filters. IEEE Transactions on Circuits and Systems 1988; 35(6):648-658.

[2] Chua LO, Lin T. Fractal pattern of second-order non-linear digital filters: a new symbolic analysis. International Journal of Circuit Theory and Applications 1990; 18:541-550.

[3] Galias Z, Ogorzalek MJ. On symbolic dynamics of a chaotic second-order digital filter. International Journal of Circuit Theory and Applications 1992; 20:401-409.

[4] Wu CW, Chua LO. Properties of admissible symbolic sequences in a second-order digital filter with overflow non-linearity. International Journal of Circuit Theory and Applications 1993; 21:299-307.

[5] Kocarev L, Chua LO. On chaos in digital filters: case $b=-1$. IEEE Transactions on Circuits and Systems-II: Analog and Digital Signal Processing 1993; 40(6):404-407.

[6] Kocarev L, Wu CW, Chua LO. Complex behavior in digital filters with overflow nonlinearity: analytical results. IEEE Transactions on Circuits and Systems-II: Analog and Digital Signal Processing 1996; 43(3):234-246.

[7] Yu X, Galias Z. Periodic behaviors in a digital filter with two's complement arithmetic. IEEE Transactions on Circuits and Systems-I: Fundamental Theory and Applications 2001; 48(10):1177-1190. 